\title{
Implementation and Promotion of Quality Control Circle: A Starter for Quality Improvement in Chinese Hospitals
}

This article was published in the following Dove Press journal: Risk Management and Healthcare Policy

\section{Dan Zhang \\ Meixia Liao \\ Tingfang Liu}

Institute for Hospital Management, Tsinghua Shenzhen International Graduate School, Tsinghua University, Shenzhen, People's Republic of China

Correspondence: Tingfang Liu Institute for Hospital Management, Tsinghua Shenzhen International Graduate School, Tsinghua University, Nanshan District, Shenzhen 518055,

People's Republic of China

$\mathrm{Tel} / \mathrm{Fax}+86-755-26036425$

Email liutf@mail.tsinghua.edu.cn
Purpose: To analyse the quality control circle (QCC) implementation status, tool modification and promotion in hospitals in mainland China.

Methods: Data were collected from the 2013-2018 National Quality Control Circle Competition of Chinese Hospitals. A total of 1913 participating QCCs implemented by 34,023 hospital staff from 915 hospitals across 31 provinces and municipalities were included in this study to analyse the characteristics of QCC implementation status in mainland China, using descriptive analyses.

Results: The majority of participating QCCs came from central and eastern hospitals. Most of the QCCs were carried out in tertiary hospitals and focused on themes of safety and patient care. The QCC has been modified in terms of its classification, implementation process, participation mechanism, and ways of dissemination. A series of promotion strategies have been made to promote the implementation of QCCs nationwide, including establishing a professional promotion organization, developing standardized training programmes, and organizing QCC academic events.

Conclusion: After years of promotion, QCCs are widely used in hospitals and have been modified to fit the practice of healthcare institutions. The QCC promotion strategies in China can offer valuable insights for other countries that are also making efforts to continuously improve care quality in healthcare institutions.

Keywords: quality control circle, promotion strategy, quality improvement

\section{Introduction}

Continuously improving service quality has been an important issue for medical institutions. The quality control circle (QCC) is viewed as a powerful tool for quality improvement. ${ }^{1}$ A QCC is a voluntary gathering of a group of employees in the same or similar work areas who meet regularly to identify, analyse and solve work-related problems by integrating qualitative and quantitative research methods. ${ }^{2}$ A QCC encourages the full participation of staff from the top managers to ordinary workers and fosters active cooperation across all levels of the organization. $^{3}$

The QCC emerged in the Japanese industry in the early 1960s and has been a commonly used technique to improve the performance of organizations and the motivation of employees. ${ }^{4}$ The QCC can be traced back to the seminars given to Japanese industrialists by Dr. W. E. Deming and Dr. J. M. Juran in the 1950 s. $^{5}$ The concept was later modified and formalized by other Japanese quality control 
experts, such as Kaoru Ishikawa. ${ }^{5}$ The QCC was introduced into mainland China from Japan in the late 1970s and has been widely adopted in the industrial sector. ${ }^{6,7}$ The QCC has been expanded into hospitals in Taiwan since 1981, but until 2008 the QCC was introduced into hospitals in mainland China. ${ }^{7-9}$ Since its introduction, the QCC has quickly become one of the most widely used quality management $(\mathrm{QM})$ tools in mainland China. A series of events has contributed to its widespread adoption. In 2011, the Ministry of Health issued the Accreditation Standards for Tertiary Comprehensive Hospitals (2011 Edition), which required hospitals to use QM tools to improve health care services for the public. ${ }^{10}$ In 2013, the China Federation for Hospital Quality Control Circle (CFHQCC) was established, and has been hosting the National Quality Control Circle Competition of Chinese Hospitals (NQCCH) annually since 2013. In 2016, the National Health and Family Planning Commission issued the Healthcare Quality Management Regulation, which emphasizes the use of QM tools such as the QCC to carry out continuous quality improvement (CQI) activities. ${ }^{11}$ In 2018, the First International Medical Quality and Safety Forum \& QCC Competition was held in China, symbolizing that QCC activities in China became international. To date, the QCC has become an iconic QM tool and has contributed to significant improvements in the quality management in China's hospitals. The promotion experience of QCCs in China can offer significant insights for other countries that are also making efforts to improving hospital quality. Therefore, this study aims to analyse and summarize the QCC implementation status, its modification and localization, and key practices of QCC promotion in hospitals in mainland China.

\section{Materials and Methods}

Data were collected from the 2013-2018 NQCCH, an academic competition annually held by the CFHQCC. The QCC teams that enter the finals of the NQCCH are required to submit detailed reports and registration information and present their QCCs onsite. The QCCs are evaluated and scored based on their submitted reports and onsite presentation. With the submitted materials and information, the CFHQCC establishes a dataset that contains the following information of participating QCCs: QCC title, QCC classification, QCC theme, number of published papers, number of patents, number of awards, other achievements, province, hospital tier, hospital type, and the leading department. A total of 1913 QCCs that participated in the finals of the NQCCH were included in this study to analyse the characteristics of the QCC implementation status in China's hospitals, using descriptive analyses. These QCCs were implemented by 34,023 hospital staff from 915 hospitals in 31 provinces and municipalities. Microsoft Excel 2016 and SPSS 20.0 to perform the data processing and statistical analysis.

\section{Results}

\section{The Status of QCC Activities in Hospitals in Mainland China}

Table 1 shows the overview of the QCCs that participated in the 2013-2018 NQCCH. The majority of the participating QCCs in the NQCCH came from tertiary hospitals $(81.7 \%)$ and public hospitals $(95.7 \%)$. Approximately $65.0 \%$ of the QCCs were carried out by nursing departments. Problem solving-oriented QCCs accounted for $93.3 \%$ of the participating QCCs.

\section{The Number of QCCs and Participants}

From 2013 to 2018, the total number of participating QCCs increased from 57 to 1457 , and the number of participants increased from 497 to 10,199. Since the second NQCCH, preliminary QCC competitions at the provincial and municipal levels have been organized in some provinces and cities, which attracted more hospital staff to engage in QCC activities. The growing number of participating QCCs and participants suggests that QCCs become increasingly popular in China's hospitals.

\section{Participating Hospitals}

Because some of hospitals participated in the NQCCH more than once, duplicated records were excluded, yielding a total of 915 unique hospitals. Figure 1 shows the number of participating QCCs in the 2013-2018 NQCCH by province-level divisions. The majority of participating QCCs came from the central and eastern China. The provinces with the most participating QCCs were Jiangsu, Jilin, Shanghai, Sichuan and Henan. These provinces all held province-level QCC competitions from 2014 to 2018, which played a positive role in promoting QCCs in these areas. Of the 1913 participating QCCs, 1562, 334 and 17 were from tertiary hospitals, secondary hospitals, and community healthcare centres, respectively, and 83 QCCs were from private 
Table I The QCCs in the 2013-2016 NQCCH $(\mathrm{N}=1913)$

\begin{tabular}{|c|c|c|c|c|c|c|c|}
\hline & Ist $\mathrm{NQCCH}$ & 2nd NQCCH & 3 rd NQCCH & 4th NQCCH & 5th NQCCH & 6th NQCCH & \multirow[t]{3}{*}{ Total } \\
\hline & in 2013 & in 2014 & in 2015 & in 2016 & in 2017 & in 2018 & \\
\hline & n (\%) & n (\%) & n (\%) & n (\%) & n (\%) & n (\%) & \\
\hline QCCs participating the $\mathrm{NQCCH}$ & 57 & 321 & 712 & 933 & 1205 & 1457 & 4685 \\
\hline QCCs entering the finals of $\mathrm{NQCCH}$ & 57 & 261 & 325 & 402 & 422 & 446 & 1913 \\
\hline Participants & 497 & 2088 & 5340 & 7464 & 8435 & 10,199 & 34,023 \\
\hline \multicolumn{8}{|l|}{ Region } \\
\hline Eastern China & 33 (57.9\%) & $142(54.4 \%)$ & 161 (49.5\%) & $189(47.0 \%)$ & $192(45.5 \%)$ & 206 (46.2\%) & $923(48.2 \%)$ \\
\hline Central China & $19(33.3 \%)$ & $86(33.0 \%)$ & 107 (32.9\%) & $130(32.3 \%)$ & $150(35.5 \%)$ & $148(33.2 \%)$ & $640(33.5 \%)$ \\
\hline Western China & $5(8.8 \%)$ & $33(12.6 \%)$ & $57(17.5 \%)$ & $83(20.6 \%)$ & $80(19.0 \%)$ & $92(20.6 \%)$ & $350(18.3 \%)$ \\
\hline \multicolumn{8}{|l|}{ Hospital tier } \\
\hline Tertiary hospital & 52 (91.2\%) & $229(87.7 \%)$ & $271(83.4 \%)$ & $326(81.1 \%)$ & $332(78.7 \%)$ & $352(78.9 \%)$ & $1562(81.7 \%)$ \\
\hline Secondary hospital & $5(8.8 \%)$ & $32(12.3 \%)$ & $52(16.0 \%)$ & 72 (17.9\%) & 85 (20.1\%) & 88 (19.7\%) & 334 (17.5\%) \\
\hline Community healthcare centre & 0 & 0 & $2(0.6 \%)$ & $4(1.0 \%)$ & $5(1.2 \%)$ & $6(1.3 \%)$ & 17 (0.9\%) \\
\hline \multicolumn{8}{|l|}{ Hospital type } \\
\hline Public hospital & $57(100.0 \%)$ & 257 (98.5\%) & 315 (96.9\%) & 385 (95.8\%) & $400(94.8 \%)$ & $416(93.3 \%)$ & $1830(95.7 \%)$ \\
\hline Private hospital & 0 & $4(1.5 \%)$ & $10(3.1 \%)$ & $17(4.2 \%)$ & $22(5.2 \%)$ & $30(6.7 \%)$ & $83(4.3 \%)$ \\
\hline \multicolumn{8}{|l|}{ QCC classification } \\
\hline Problem solving-oriented QCC & $57(100.0 \%)$ & $261(100.0 \%)$ & $322(99.1 \%)$ & $382(95.0 \%)$ & $373(88.4 \%)$ & $390(87.4 \%)$ & $1785(93.3 \%)$ \\
\hline Research project-oriented QCC & 0 & 0 & $3(0.9 \%)$ & $20(5.0 \%)$ & 49 (11.6\%) & $56(12.6 \%)$ & $128(6.7 \%)$ \\
\hline \multicolumn{8}{|l|}{ Leading department } \\
\hline Nursing & $35(61.4 \%)$ & 207 (79.3\%) & $223(68.6 \%)$ & $244(60.7 \%)$ & $263(62.3 \%)$ & $27 \mid(60.8 \%)$ & $1243(65.0 \%)$ \\
\hline Medical technological & $9(15.8 \%)$ & $25(9.6 \%)$ & $4 \mid(12.6 \%)$ & 64 (15.9\%) & 37 (8.8\%) & 38 (8.5\%) & $214(11.2 \%)$ \\
\hline Administrative and logistic & $2(3.5 \%)$ & $6(2.3 \%)$ & $9(2.8 \%)$ & $24(6.0 \%)$ & $16(3.8 \%)$ & $21(4.7 \%)$ & $78(4.1 \%)$ \\
\hline Clinical & $8(14.0 \%)$ & $13(5.0 \%)$ & $34(10.5 \%)$ & $45(11.2 \%)$ & $71(16.8 \%)$ & $76(17.0 \%)$ & 247 (12.9\%) \\
\hline Pharmaceutical & $2(3.5 \%)$ & $7(2.7 \%)$ & $11(3.4 \%)$ & $13(3.2 \%)$ & $20(4.7 \%)$ & $25(5.6 \%)$ & $78(4.1 \%)$ \\
\hline Others & $\mathrm{I}(1.8 \%)$ & $3(1.1 \%)$ & $7(2.2 \%)$ & $12(3.0 \%)$ & $15(3.6 \%)$ & $15(3.4 \%)$ & $53(2.7 \%)$ \\
\hline \multicolumn{8}{|l|}{ QCC theme } \\
\hline Safety & $20(35.1 \%)$ & 99 (37.9\%) & $112(34.5 \%)$ & 112 (27.9\%) & $109(25.8 \%)$ & $113(25.3 \%)$ & 565 (29.5\%) \\
\hline Patient care & $11(19.3 \%)$ & 52 (19.9\%) & $70(21.5 \%)$ & 97 (24.1\%) & 101 (23.9\%) & $104(23.3 \%)$ & 435 (22.7\%) \\
\hline Time efficiency & $10(17.5 \%)$ & $34(13.0 \%)$ & $36(11.1 \%)$ & $67(16.7 \%)$ & $64(15.2 \%)$ & $68(15.2 \%)$ & $279(14.6 \%)$ \\
\hline Administrative management & $4(7.0 \%)$ & $22(8.4 \%)$ & $20(6.2 \%)$ & 45 (11.2\%) & $57(13.5 \%)$ & $60(13.5 \%)$ & $208(10.9 \%)$ \\
\hline Cost management & $2(3.5 \%)$ & $14(5.4 \%)$ & $27(8.3 \%)$ & $19(4.7 \%)$ & $23(5.5 \%)$ & $25(5.6 \%)$ & $110(5.8 \%)$ \\
\hline Medical technology & $2(3.5 \%)$ & $7(2.7 \%)$ & $20(6.2 \%)$ & $33(8.2 \%)$ & $38(9.0 \%)$ & $43(9.6 \%)$ & $143(7.5 \%)$ \\
\hline Education and training & $5(8.8 \%)$ & $14(5.4 \%)$ & $22(6.8 \%)$ & $9(2.2 \%)$ & $14(3.3 \%)$ & $15(3.4 \%)$ & $79(4.1 \%)$ \\
\hline Satisfaction & $2(3.5 \%)$ & $16(6.1 \%)$ & II (3.4\%) & II (2.7\%) & $9(2.1 \%)$ & $10(2.2 \%)$ & $59(3.1 \%)$ \\
\hline Documentation and record & $\mathrm{I}(1.8 \%)$ & $3(1.1 \%)$ & $7(2.2 \%)$ & $9(2.2 \%)$ & $7(1.7 \%)$ & $8(1.8 \%)$ & 35 (1.8\%) \\
\hline \multicolumn{8}{|l|}{ Achievement } \\
\hline Number of published papers & 11 & 89 & 161 & 275 & 351 & 393 & 1280 \\
\hline Number of patents & 0 & 9 & 32 & 46 & 53 & 66 & 206 \\
\hline Number of awards & 0 & 5 & 17 & 28 & 37 & 45 & 132 \\
\hline
\end{tabular}

hospitals. The variety of hospital levels and types suggests that QCCs have been widely used by different types of healthcare institutions in China.

\section{Participating Departments}

The majority of participating QCCs were led by nursing departments $(65.0 \%)$, followed by clinical departments (12.9\%) and medical technological departments (11.2\%). In terms of QCC classification, $40.9 \%$ of QCCs led by clinical departments were research project-oriented QCCs, while 72.5\% of QCCs led by nursing departments were problem solving-oriented QCCs. 


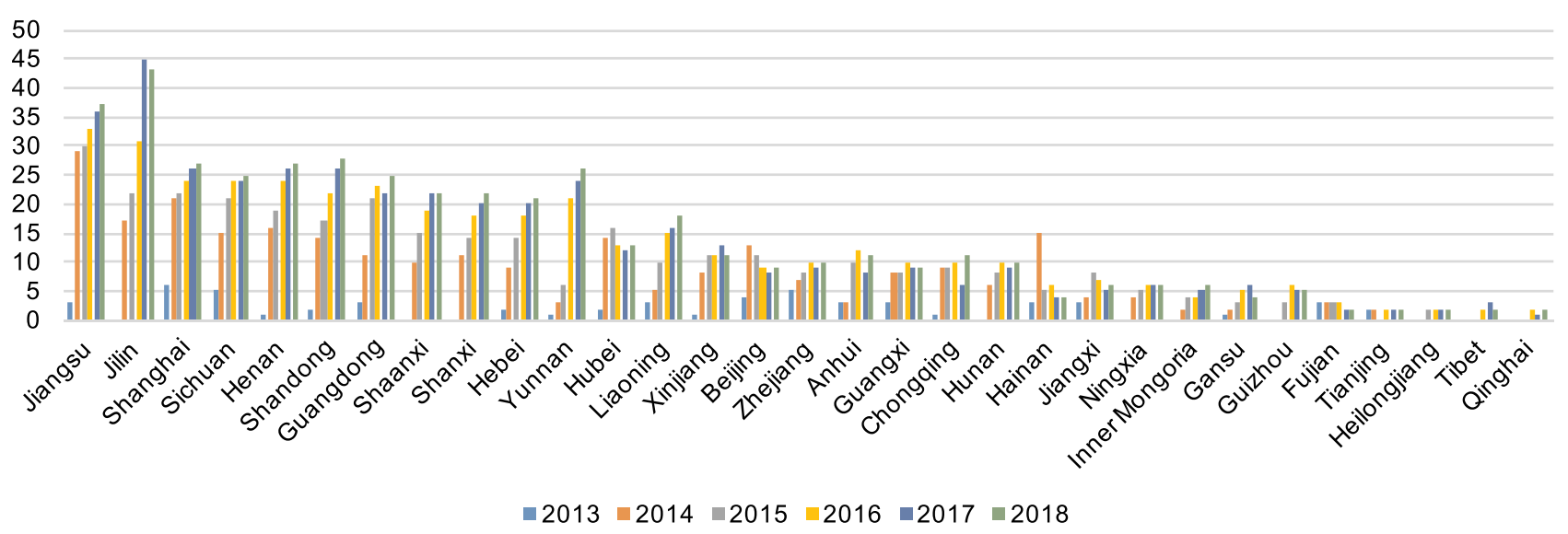

Figure I The number of participating QCCs in the 2013-2018 NQCCH by province-level divisions.

\section{The Themes of QCCs}

The themes of participating QCCs can be classified into safety, patient care, time efficiency, administrative management, cost management, medical technology, education and training, documentation and record, and satisfaction. Most of the participating QCCs focused on the themes of safety (29.5\%) and patient care (22.7\%). The top three issues of the participating QCCs were reducing the incidence of falls, reducing the incidence of pressure ulcers and reducing pharmacy dispensing errors.

\section{QCC Achievements}

The numbers of published papers, patents and awards have increased over time. Prior to participating in the NQCCH, some QCCs won awards that were given by other institutions or organizations. A total of 132 awards were obtained by 1913 participating QCCs. In total, 1280 QCC-related academic papers have been published in journals, and 206 patents have been obtained from participating QCCs. Based on the literature search in the China National Knowledge Infrastructure (CNKI) database of references and abstracts, more than 1000 QCC-related publications have been published each year since 2014, as shown in Figure 2.

\section{The Modification and Localization of QCC}

The practice of QCCs in China's hospitals has been beyond its original concept and scope. The practice integrated the ideas of LEAN, quality function deployment (QFD), and other QM tools, and has been modified and adapted to China's environmental context by developing a set of QCC processes and mechanisms that are suitable for healthcare institutions.

\section{The Modification of QCC Classification and Implementation Process}

Generally, based on the themes of QCCs, the QCCs in industry can be classified into five types: manufacturing process-related QCCs, service-related QCCs, technologyrelated QCCs, management-related QCCs and innovative QCCs. ${ }^{12}$ Based on the complexity of the issues and objectives, the QCCs in health care are classified into only two types: problem solving-oriented QCCs and research projectoriented QCCs. A decision table for QCC classification has been designed to help participants determine the type of QCCs, as shown in Table 2. Problem solving-oriented QCCs focus on a specific and individual issue and aim to maintain or improve the current situation by exploring and eliminating the causes of the issue. Research project-oriented QCCs focus on systemic problems and aim to generate innovation-infused solutions by adopting scientific thinking and systematic perspective. These two types of QCCs are also different in terms of their theoretical foundation. Problem solving-oriented QCCs are based on plan-do-checkact (PDCA), while research project-oriented QCCs are based on plan-do-study-act (PDSA). PDSA is considered more effective than PDCA, as it includes the Study stage that goes beyond mere checking whether improvements are made, but critically reflecting on how and why improvements have been made. An example of a problem solving-oriented QCC is the reduction of the rate of surgical site infection, while an example of a research project-oriented QCC is the establishment of a hospital-community-family-based model for cervical spondylosis prevention and treatment.

In terms of implementation process, both types of QCCs are implemented through four stages and 10 steps, as shown in Figure 3. The implementation process of QCCs in health 


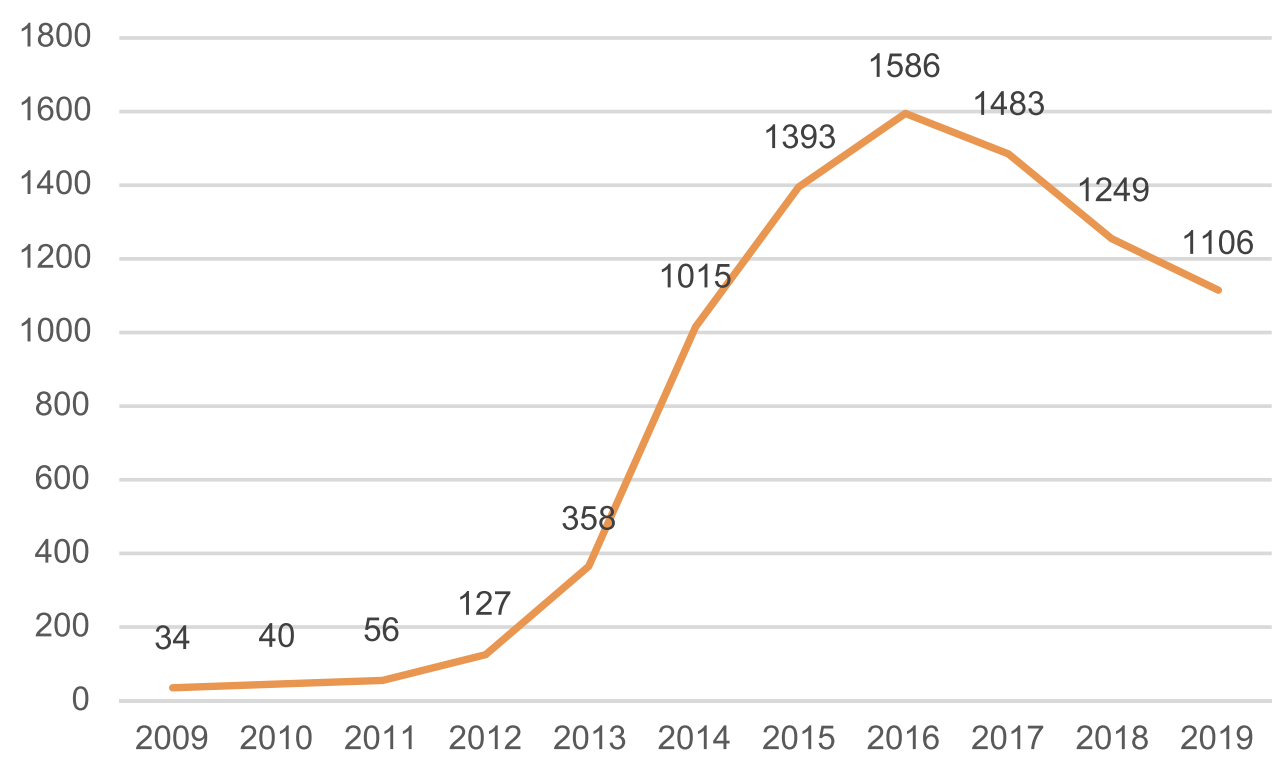

Figure 2 Number of QCC-related publications in China National Knowledge Infrastructure (CNKI).

care is slightly different from that of QCCs in other industries. Generally, steps for setting up QCCs in industrial sectors consist of the following eight steps: identification of a problem, status analysis of the problem, goal setting, cause effect analysis, implementation of solution, result confirmation, standardization, and review. ${ }^{12}$ Problem solving-oriented QCCs share a similar implementation process with the QCCs in industrial sectors but include the steps of developing an action plan and generating solutions in addition to the above eight steps. An action plan enables circle members to monitor their progress and take each task step-by-step, therefore increasing the efficiency and effectiveness. The step of generating solutions emphasizes the formulation, assessment and planning of the solutions to identify appropriate solutions and efficient implementation process.

By integrating scientific research approaches, the implementation process of research project-oriented QCCs is different from that of problem solving-oriented QCCs in terms of the following steps. In the step of theme selection, it is necessary to define research objectives and the scope and conduct a comprehensive literature review. If the problem can be solved by existing literature or empirical findings, it is inappropriate to launch a research project-oriented QCC. In the planning step, circle members need to develop a technology roadmap that can help them to identify key issues and solutions in a more holistic way. In the step of identifying appropriate solutions, PDPCs should be used to anticipate any obstacles and problems that might surface in the implementation of each solution, and pre-tests should be conducted to verify the effectiveness of each solution. In the step of effect confirmation, the effectiveness of solutions should be evaluated by controlled trials. The introduction of a scientific approach in QCCs has greatly improved the quality and output of QCC activities. One research projectoriented QCC usually can produce a number of research articles, guidelines or patents.

\section{The Modification of Participation Mechanism}

The traditional QCC activities in industry emphasize on the involvement and contributions of the circle members who do the same or similar work. Given that the delivery of healthcare services usually requires intra-departmental teamwork, the participation mechanism of QCCs have been modified to fit the need for interprofessional cooperation in healthcare institutions. On one hand, the circle members usually consist of hospital staff from different departments. For example, besides physicians, the circle members of a QCC led by the clinical department can consist of pharmacists, information engineers, and administrative staff. On the other hand, the QCCs in China's hospitals have established a mechanism that allows the participation of non-members, thereby transferring from a closed circle to an open circle. Under this mechanism, non-members may be invited to present their ideas during the implementation process of QCCs, such as the process of theme selection, goal setting, cause analysis, solution formulation and standardization. Such an open mechanism allows wider participation within the organization and brings greater benefits. 
Table 2 The Decision Table for QCC Classification

\begin{tabular}{|c|c|c|}
\hline $\begin{array}{l}\text { Research Project- } \\
\text { Oriented QCCs }\end{array}$ & $\begin{array}{l}\text { Relevance } \\
\text { Highly } \\
\text { Relevant: } \\
2 \text { Points; } \\
\text { Relevant: } \\
\text { I Point; } \\
\text { Not } \\
\text { Relevant: } \\
\text { O Point }\end{array}$ & $\begin{array}{l}\text { Problem Solving- } \\
\text { Oriented QCCs }\end{array}$ \\
\hline $\begin{array}{l}\text { Aim to solve a new or } \\
\text { systematic problem }\end{array}$ & & $\begin{array}{l}\text { Aim to solve an existing } \\
\text { problem }\end{array}$ \\
\hline $\begin{array}{l}\text { Aim to greatly improve } \\
\text { the current situation or } \\
\text { introduce innovation }\end{array}$ & & $\begin{array}{l}\text { Aim to maintain or } \\
\text { improve the current } \\
\text { situation }\end{array}$ \\
\hline $\begin{array}{l}\text { Aim to meet a high } \\
\text { standard of excellence } \\
\text { and quality }\end{array}$ & & $\begin{array}{l}\text { Aim to maintain current } \\
\text { quality level }\end{array}$ \\
\hline $\begin{array}{l}\text { Aim to prevent } \\
\text { a foreseeable problem or } \\
\text { issue }\end{array}$ & & $\begin{array}{l}\text { Aim to prevent an } \\
\text { existing problem }\end{array}$ \\
\hline $\begin{array}{l}\text { Goals can be achieved by } \\
\text { exploring and } \\
\text { implementing innovation- } \\
\text { infused solutions }\end{array}$ & & $\begin{array}{l}\text { Goals can be achieved by } \\
\text { exploring and eliminating } \\
\text { the causes of the issue }\end{array}$ \\
\hline Total score & & Total score \\
\hline Adopt/Not adopt & & Adopt/Not adopt \\
\hline
\end{tabular}

\section{The Establishment of Dissemination Mechanism for QCC Findings}

In order to bring greater benefits, there are several ways to disseminate findings and successful solutions from QCCs. After the completion of QCCs, circle members usually make a presentation of their findings to other staff in their hospitals. Excellent QCCs can participate in province-level or national-level QCC competitions to further disseminate their findings. Furthermore, academic articles can be written for publication in journals. In order to empower participants to implement QCCs in a standardized manner, detailed QCC practice guidelines have been formulated and issued by the CFHQCC. The guidelines have been revised six times, with the first edition emphasizing the proper use of tools and techniques and the sixth edition prioritizing the significance and innovation of the QCCs. Continuously updated guidelines enable circle members to implement QCCs in accordance with the latest concepts and best practices.

\section{Discussion}

\section{The Status of QCC Activities in Hospitals in Mainland China}

China has the largest number of hospitals in the world. As of the end of April 2020, there were 34,464 hospitals in mainland China. ${ }^{13}$ Our study shows that more than 900 hospitals participated in the finals of the NQCCH. The growing number of participating QCCs and participants suggests that QCCs have become increasingly popular across China. According to the estimation of the CFHQCC, there have been approximately 60,000 QCCs launched by hospitals in mainland China, with approximately one million hospital staff nationwide participating in QCC activities. The following three factors may explain their widespread adoption. First, the government emphasizes on the use of QM tools to continuously improve the quality of care in hospitals by issuing the Accreditation Standards for Tertiary Comprehensive Hospitals and the Healthcare Quality Management Regulation. Second, the modification and localization of QCCs improve the feasibility of QCCs in health care. Third, the CFHQCC promotes the use of QCCs by developing and offering standardized training programmes, organizing QCC academic events, and engaging in international cooperation and exchange.

Our results show that more QCCs were implemented in hospitals in the central and eastern China. This is probably because the local governmental health agencies in these regions value QCC activities and hold province-level QCC competitions. Furthermore, the central and eastern China enjoy more economic prosperity and medical resources. As a result, hospitals in these regions are better managed and more receptive to novel managerial tools. Our analysis also suggests that the majority of QCCs were carried out in tertiary hospitals. This may be attributed to the fact that adopting the ideas and tools of CQI is one of the criteria in the Accreditation Standards for Tertiary Comprehensive Hospitals and the QCC becomes a commonly used QM tool due to its higher feasibility.

Nurses and physicians play a different leading role in problem solving-oriented QCCs and research projectoriented QCCs. Problem solving-oriented QCCs are easier to learn and implement than research project-oriented QCCs. Accordingly, nurses become the major force in the use of problem solving-oriented QCCs. In contrast, research project-oriented QCCs focus on systemic problems that usually require more rigorous research. Physicians who 


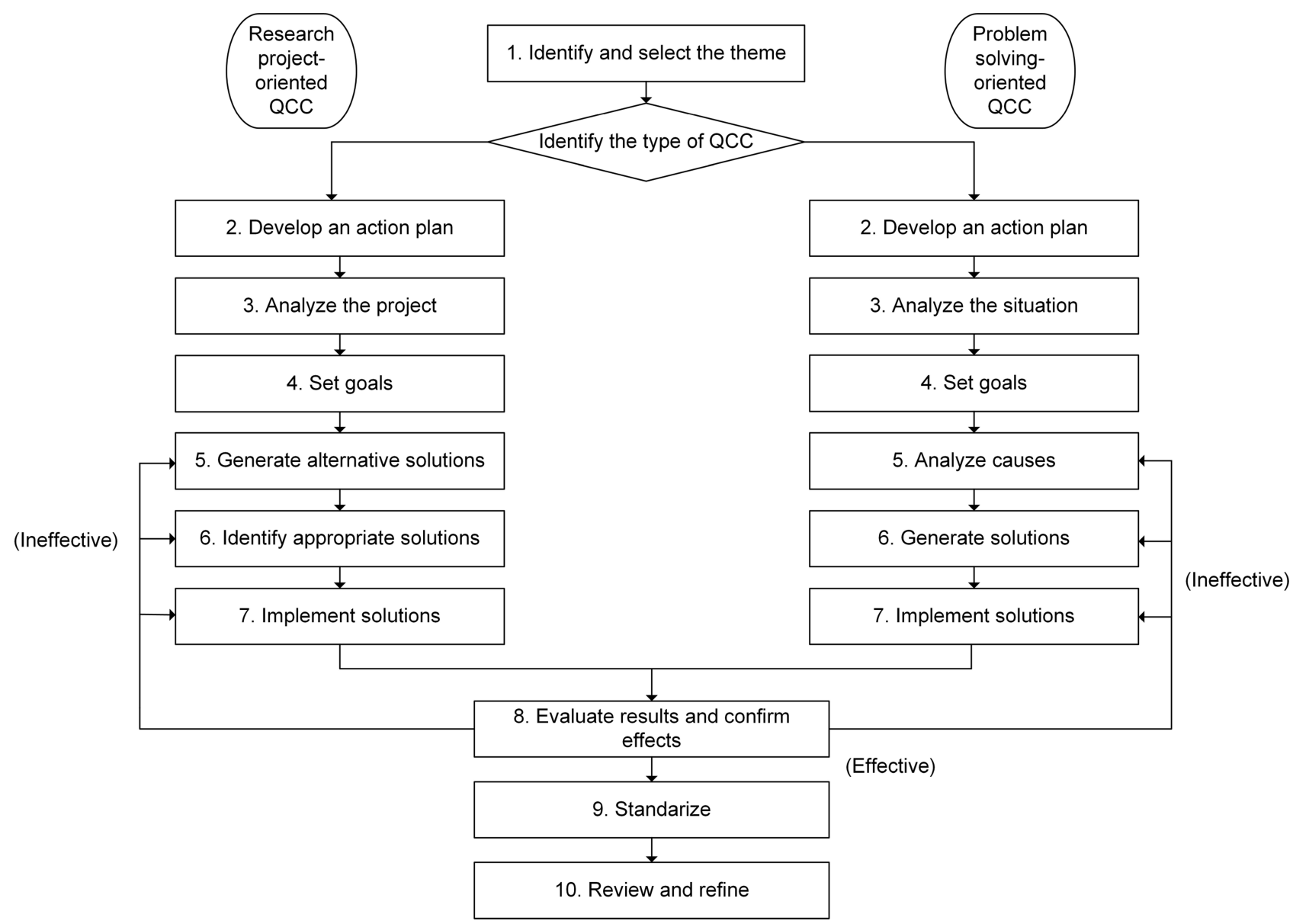

Figure 3 The implementation process of problem solving-oriented QCC and research project-oriented QCC.

usually have profound scientific ability are more used to the scientific thinking and scientific approach embedded in research project-oriented QCCs. As a result, most of the research project-oriented QCCs were led and carried out by physicians. Nonetheless, both types of QCCs require interprofessional cooperation of different departments.

In terms of QCC themes, most of the QCCs focused on safety and patient care. In China, healthcare institutions have paid growing attention to patient-centred care, resulting in more QCCs related to patient care and patient safety. In addition, some QCCs focus on the safety of hospital staff, such as physician safety and protection in the outpatient setting, and stress management for hospital staff.

Previous literatures suggest that the QCC used in health care is likely to be a powerful technique for facilitating high-quality healthcare and improved outcomes. ${ }^{14-19}$ The study by Wang et $\mathrm{al}^{14}$ reviewed the tangible and intangible achievements of 92 QCCs carried out in a medical institution in Zhejiang province. This study showed that QCCs improve performance indicators in terms of reducing costs and internal errors, and improving work quality, patient satisfaction and economic performance. Reported intangible benefits include developing problem-solving capability, promoting communication and coordination, increasing self-confidence, and developing a greater sense of responsibility. The study by Zhang et $\mathrm{al}^{15}$ indicated that QCCs can play an active role in enhancing hospital staff's perceptions of patient safety culture. Previous studies also suggest that elements of QCCs such as audit and feedback, workshops, and educational materials can positively result in changes in the behaviour of healthcare professionals, which contributes to improved individual and group performance. ${ }^{16,17}$ Our study shows that QCC activities can facilitate academic achievements, such as academic papers and patents, indicating that QCCs may benefit hospital staff with improved scientific research abilities. Therefore, the wide adoption of QCCs in China's hospitals are likely to have brought quality improvement to hospitals and continuing professional development to hospital staff. 


\section{The Necessity of QCC Modification and Localization}

A great number of management tools used in the health care are borrowed from industrial sectors. However, there are differences between healthcare institutions and industrial enterprises. Healthcare delivery requires interprofessional collaboration, and its delivery process demands both standardization and personalization. These differences make it necessary to modify these management tools when applying them in health care. Research project-oriented QCCs combine quality management with scientific research, which makes it suitable for health professionals with profound scientific ability and greatly improves the quality and value of QCC activities. The detailed implementation process provides guidance on how to implement QCC activities in hospitals in a standardized manner. The circle members from diverse departments fit the need for interprofessional cooperation in healthcare institution and the mechanism of non-member participation allows more personnel to engage in QCC activities. These modification and localization of QCCs make the QCC more applicable to healthcare institutions.

\section{The Experience and Strategies of QCC Promotion in Mainland China}

The above analysis shows that QCCs have gained wide popularity in China's hospitals and great achievement in terms of their quantity and quality. After years of practice and promotion, the QCC has been modified to fit the practice of healthcare institutions. The successful promotion of QCCs in China's hospitals can offer valuable insights for other countries. Therefore, the following section summarizes the key practices of QCC promotion in mainland China.

\section{Establishing a Professional Promotion Organization}

To promote the use of QCCs in hospitals, the CFHQCC, a nationwide academic, non-profit organization, was established in 2013, which is dedicated to promoting the ideas and tools of CQI in hospitals and establishing a platform where hospital managers can learn and share QCC activities. To promote the application of other QM tools in hospitals, the CFHQCC was renamed as the China Federation for Hospital Quality Management (CFHQM) in 2017 and established eight specialized study groups for eight QM tools, such as tracer methodology, root cause analysis (RCA), and healthcare failure mode and effect analysis (HFMEA). The CFHQM has built a website where free online learning resources are available to the public. Associations have been established in some provinces and cities and greatly promoted the widespread application of QCCs in mainland China.

\section{Developing and Offering Standardized Training Programmes}

An effective training programme is necessary to ensure that the personnel involved in QCCs have the necessary knowledge and skills. The CFHQM has developed detailed standard operating procedures and guidelines for implementing QCCs, provided benchmark QCC cases to empower participants to design, implement and lead QCC activities in a standardized manner, and offered free training programmes to further promote ideas and knowledge about QCC. During 2015 and 2019, the CFHQM has offered free training programmes such as advanced training programmes for tertiary hospitals, with approximately 1500 participants enrolling in training programmes every year.

\section{Organizing QCC Academic Events}

Since its establishment, the CFHQM has organized a series of academic events to promote the use of QCCs in mainland China, among which the NQCCH is the most influential. The NQCCH has played a significant role in raising the awareness of QM and encouraging the use of QM tools in hospitals. In terms of the competition rules, there are two rounds of competitions: the preliminary round and the final round. In the preliminary round, the participating QCCs are evaluated based on their written materials. In the final round, the QCCs are evaluated based on their onsite presentation. To ensure the justice and fairness of competition, several independent sessions are held in the final round according to the hospital level and QCC classification. Most judges are experts from the CFHQM, and the judges for each competition are chosen at random. In terms of the award setting, the NQCCH has set up as many awards as possible to encourage more participations. In addition to the participants, audiences are welcomed to the NQCCH. In terms of the participants, $45 \%$ are audiences who have neither participated in the competition nor entered the final round. By observing the onsite presentations of the participating QCCs, these observers can learn the QCC knowledge and skills, as well as solutions and experiences of excellent QCC cases. 
Since 2015, the CFHQM has been holding the annual National Forum on Quality Management Tools in Hospitals. The forum creates a platform where hospital managers can share the ways to promote QM tools. Hospitals with successful QCC practices will be invited to share their experiences with promotion or regulation mechanisms. Hospitals with successful practice of other QM tools, such as RCA, HFMEA, and LEAN, will also be invited to share their experiences. Through mutual interactions and idea exchanges, hospital managers can enhance their knowledge about leading practices related to QM tools.

The CFHQM has also actively been engaged in international cooperation and exchange. The CFHQM reached a comprehensive strategic cooperation with the International Society for Quality in Health Care (ISQua), and they jointly organized the First International Medical Quality and Safety Forum \& QCC Competition in 2018 in China. In the ISQua's First Quality Competition held in 2019, the Chinese team won the first prize, and the scoring scheme developed by the CFHQM was adopted by this competition. These international competitions provide a platform for QCC participants to share their best practice experiences and lessons to other countries. These international exchange opportunities also stimulate more hospital staff to participate in QCCs.

\section{Implications}

The continuous improvement of healthcare quality achieved through QM tools has been an important driving force for the long-term development of healthcare institutions. The abovementioned experiences and strategies of QCC promotion in hospitals in mainland China can offer important insights for other countries and regions that are considering the adoption of QCCs to improve the quality of their healthcare institutions. This information can also be valuable for the promotion of other quality management tools in China and other countries. In fact, the CFHQM has adopted similar strategies to promote the application of other quality tools, such as RCA, HFMEA, QFD, LEAN and Six Sigma. These promotion practices have been advancing very well, suggesting that these promotion strategies are effective.

\section{Limitations}

A number of limitations should be considered regarding this study. The data analysis in this study was based on the datasets of the 2013-2016 NQCCH, which mainly allowed descriptive analysis. In addition, given that this study only included QCCs that have entered into the finals for data analysis and that those QCCs were usually excellent cases, the analysis results might exaggerate the actual effects of QCC promotion in mainland China. Future research is needed to further explore the problems of promotion practices and the influencing factors of implementation effects through field surveys and questionnaire surveys.

\section{Conclusion}

This study analysed the QCC implementation status, QCC modification and localization, and promotion practices at hospitals in mainland China. After years of promotion, the QCC has become a widely used QM tool in China's hospitals. The modification and localization have been made to fit the practice of healthcare institutions. A series of promotion strategies have contributed to the implementation of QCCs in hospitals nationwide, including establishing a professional promotion organization, offering standardized training programmes, and organizing QCC academic events. These practices and strategies can offer valuable insights for other countries and regions and for the promotion of other QM tools.

\section{Data Sharing Statement}

The data that support the findings of this study are available from China Federation for Hospital Quality Management. Data are available to the corresponding author with the permission of China Federation for Hospital Quality Management.

\section{Ethical Approval}

The data that support the findings of this study are originally collected by China Federation for Hospital Quality Management. This study conducted the data analysis with the permission of China Federation for Hospital Quality Management and maintained the confidentiality of the data. As this study does not contain any studies with human participants performed by any of the authors, it does not require formal ethical approval.

\section{Acknowledgments}

The authors are grateful to the support of the China Federation for Hospital Quality Management. The funders had no role in the study design, data analysis, or manuscript writing.

\section{Funding}

This work was supported by the Research Fund of Shenzhen Public Hospital Management Center [grant number 20199660001]; and the Young Teacher Research Fund of Graduate School at Shenzhen, Tsinghua 
University [grant number 20195720011]. This work was also a project of the Shenzhen Key Research Base of Humanities and Social Sciences.

\section{Disclosure}

The authors report no conflicts of interest in this work.

\section{References}

1. Middleman RR. The quality circle: fad, fix, fiction. $A d m$ Soc Work. 1984;8:31-44. doi:10.1300/J147v08n01_03

2. Lillrank P. The transfer of management innovations from Japan. Organization Studies. 1995;16:971-989. doi:10.1177/01708406950160 0603

3. Watanabe S. The Japanese quality control circle: why it works. Int Labour Rev. 1991;130:57-80.

4. Kolchin MG. Borrowing back from the Japanese. Adv Manage J. $1887 ; 52: 26-35$.

5. Nonaka I. History of the quality circle. Qual Prog. 1993;26:81-83.

6. Ye SS, Wang DX. Quality Control Circle Activities for Service Industry. Shanghai: Fudan University Press; 2009.

7. Liu TF, Liu Y. The Quality Control Circle Handbook for Chinese Hospitals. Beijing: People's Medical Publishing House; 2012.

8. Dai YJ. Quality Management. Taipei: San Min Book Co., Ltd; 2001.

9. Zhu SD. The Quality and Patient Safety Management in Medical Institution: Theory and Practice. Taipei: Farseeing Publishing Co., Ltd; 2011.

10. Ministry of Health of the People's Republic of China. Accreditation standards for tertiary comprehensive hospitals; 2011. Available at: http://www.nhc.gov.cn/wjw/gfxwj/201304/0404f9cd71764ab29 b2365e069cfbf2d.shtml. Accessed February 16, 2020.
11. National Health and Family planning commission of china. Healthcare quality management regulation. Available at: http:// www.nhc.gov.cn/fzs/s3576/201610/ae125f28eef24ca 7 aac57 c8ec530c6d2.shtml. Accessed February 16, 2020.

12. China Association for Quality. The Quality Control Circle Handbook. Beijing: China Social Sciences Publishing House; 2008.

13. National Health Commission of the People's Republic of China. The number of healthcare institutions in China as of the end of April 2020. Available at: http://www.nhc.gov.cn/mohwsbwstjxxzx/s7967/ 202007/a365f0c05dcb456ca040c0ee5c160684.shtml. Accessed April 27, 2020.

14. Wang LR, Wang Y, Lou Y, et al. The role of quality control circles in sustained improvement of medical quality. SpringerPlus. 2013;2:141. doi:10.1186/2193-1801-2-141

15. Zhang D, Liao M, Zhou Y, Liu T. Quality control circle: a tool for enhancing perceptions of patient safety culture among hospital staff in Chinese hospitals. Int J Qual Health Care. 2020;32(1):64-70. doi:10.1093/intqhe/mzz094

16. Rohrbasser A, Mickan S, Harris J. Exploring why quality circles work in primary health care: a realist review protocol. Syst Rev. 2013;2:110. doi:10.1186/2046-4053-2-110

17. Zaher E, Ratnapalan S. Practice-based small group learning programs: systematic review. Can Fam Physician. 2012;58 (6):637-642.

18. Schmele JA, Allen ME, Butler S, Gresham D. Quality circles in the public health sector: implementation and effect. Public Health Nurs. 1991;8(3):190-195. doi:10.1111/j.1525-1446.1991. tb00754.x

19. Rohrbasser A, Harris J, Mickan S, Tal K, Wong G. Quality circles for quality improvement in primary health care: their origins, spread, effectiveness and lacunae- a scoping review. PLoS One. 2018;13 (12):e0202616. doi:10.1371/journal.pone.0202616
Risk Management and Healthcare Policy

\section{Publish your work in this journal}

Risk Management and Healthcare Policy is an international, peerreviewed, open access journal focusing on all aspects of public health, policy, and preventative measures to promote good health and improve morbidity and mortality in the population. The journal welcomes submitted papers covering original research, basic science, clinical \& epidemiological studies, reviews and evaluations, guidelines, expert opinion and commentary, case reports and extended reports. The manuscript management system is completely online and includes a very quick and fair peer-review system, which is all easy to use. Visit http://www.dovepress.com/testimonials.php to read real quotes from published authors. 